\title{
Editorial
}

Himat Vaghadia MB BS MHSC FRCPC

\section{Outpatient anesthesia. Some aging perspectives: advice from a caterpillar}

\author{
"You are old, Father William," the young man said \\ "And your hair has become very white; \\ And yet you incessantly stand on your head- \\ Do you think, at your age, it is right?" \\ (Alice's Adventures in Wonderland)
}

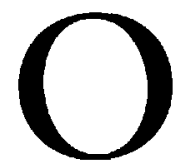

UTPATIENT anesthesia is and will continue to be a challenging and stimulating area of professional practice in the foreseeable future due to the confluence of two inexorable trends. First, there has been a shift in surgical services to an ambulatory basis for financial prudence. Secondly, the aging of 'baby boomers' continues. Surgical needs of the latter will increasingly be met in the outpatient arena. The Toronto data presented by Chung $e t a l .^{1}$ offer interesting insights into some aging perspectives.

Elderly patients do not pose an extra risk in the outpatient setting as judged by standard measures of quality (mortality and absence of serious complications) when administered monitored anesthesia care (MAC). They do experience physiological disturbances (mainly cardiovascular) consequent to the surgical trespass and their state of health, but these do not appear to harm them. Their appropriate management lies within the scope of every anesthesiologist. Certain physiological functions are inherently unstable under anesthesia because many neural reflexes that modulate these functions are impaired by anesthetics. Evaluation of the quality of an anesthetic on the presence and magnitude of changes in BP, HR and other physiological variables is interesting but is without any scientific basis. ${ }^{2} \mathrm{~A}$ link has to be clearly demonstrated between morbidity and such changes in physiology, and even if such a link were to be shown, the end (low mortality and morbidity) does not justify the means( the prohibitive cost of large trials). In fact, a link between the patient's disease state and perioperative complications is more important. ${ }^{3}$ Practical experience of the anesthesiologist is another consideration. ${ }^{3}$

The Toronto results also allow us to examine other perspectives that have challenged us for a long time. Anesthesiologists are often expected to admit that pain and vomiting are the unavoidable sequelae of their art. However, if the object of the anesthetic is to re-establish suspended functions and capabilities in an expeditious manner, the presence of pain and vomiting must place the anesthetic within the judgement of all patients. Patients continue to express a strong desire to avoid clinical outcomes such as pain and vomiting. ${ }^{4}$ Therefore, it is relevant that we assess our performance with these issues in mind. The incidence of vomiting in the Toronto series was $2.7 \%$ in the general anesthesia(GA) group and $0.6 \%$ in elderly patients who mostly received MAC. Is this acceptable? Historical comparisons (Table) will help to demonstrate that these results are excellent and, other centres should aim for a similar performance. Can this be achieved in other centres? Probably. Edsall ${ }^{5}$ has shown that quarterly CQI (continuous quality improvement) aimed at addressing PONV was successful in reducing the rate from $14 \%$ to $6 \%$ in eight months. Measures that were used included minimizing the use of narcotics and pretreatment of at risk cases.

The management of pain and the role of opioids in outpatients are other important perspectives. It is cruel to leave a patient in the recovery room with pain for want of adroitness, at the mercy of a nurse whose immediate recourse is a syringe of opioid. Before the discovery of anesthesia it was customary to prescribe laudanum to dull the pain of surgical incision, but patients vomited it uselessly into the pail. Even then, doctors were beginning to suspect that this was an effect of the opioid on the brain. Yet, today, we rely on 
TABLE Rates of PONV in outpatients from 1923 to 1998 (ether to propofol). ${ }^{1-10}$

\begin{tabular}{llll}
\hline Author (year) & $n$ & Rates of PONV (\%) & Anesthetic technique \\
\hline Lundy (1923) & 500 & 25 & Ether, $\mathrm{N}_{2} \mathrm{O}, \mathrm{O}_{2}$ \\
Brindle (1975) & 500 & 16 & Halothane, $\mathrm{N}_{2} \mathrm{O}, \mathrm{O}_{2}$ \\
Duncan (1993) & 6914 & 7 & Isoflurane, $\mathrm{N}_{2} \mathrm{O}, \mathrm{O}_{2}$ \\
Chung (1996) & 1017 & 7 & Propofol, \\
& & & Isoflurane, $\mathrm{N}_{2} \mathrm{O}, \mathrm{O}_{2}$ \\
Chung (1998) & 17638 & 2 & Propofol, Isoflurane, \\
& & & $\mathrm{N}_{2} \mathrm{O}, \mathrm{O}_{2}$ \\
\hline
\end{tabular}

the same remedy for analgesia. The Toronto series also helps to demonstrate the interaction between pain and opioids. In the younger patients who received GA, the rate of PONV in PACU was $2.7 \%$ but almost doubled in the ASU as a result of opioid administration in PACU. It should not be surprising that even propofol anesthesia has been found to be of doubtful clinical efficacy in an analysis of PONV in 84 studies involving 6069 patients. $^{6}$ The best propofol anesthetic will be rendered ineffectual if opioids are administered in the PACU. The Toronto results demonstrate that pain can be effectively controlled and frequency of pain requiring treatment in PACU can be decreased to very low levels $(6 \%)$. This is promising because poor management of pain in outpatients is a source of ongoing concern. In some outpatient units that rely exclusively on opioids, $40 \%$ of patients report moderate to severe pain during hospitalization. ${ }^{7}$ Such results are unacceptable. The Toronto results also demonstrate that MAC is associated with very low rates of pain $(0.6 \%)$ and vomiting $(0.6 \%)$ and, hence, a shorter stay in hospital and a lower risk of readmission. Anaesthesiologists are strongly encouraged to consider alternatives to opioids, such as multi-modal anesthesia employing NSAIDs and local anesthetics, in all outpatients.

In summary, it is a cruel paradox that just as patients judge a surgeon's skill by the appearance of the incision, so will they judge an anesthetic by the absence of vomiting and pain, and not by the unseen hurdles that were overcome during their insensibility. It is highly recommended that outpatient facilities need to implement strategies that employ voluntary compliance with CQI aimed at achieving the bench marks set by the Toronto series.

"In my youth", Father William replied to his son, "I feared it might injure the brain;

But, now that I'm perfectly sure I have none, Why, I do it again and again."

(Alice's Adventures in Wonderland)

\section{L'anesthésie ambula- toire. L'optique du vieillissement : conseil d'une chenille}

\author{
"Vous êtes vieux, père William, dit le jeune homme, \\ Et vos rares cheveux sont devenus très blancs; \\ Sur la tête pourtant vous restez planté comme \\ Un poirier: est-ce bien raisonnable, vraiment?" \\ (Alice au Pays des Merveilles) \\ (Traduction de Henri Parisot)
}

L'anesthésie ambulatoire est et continuera d'être dans un proche avenir un défi à relever et un domaine stimulant de la pratique professionnelle à cause de la confluence de deux inévitables tendances. D'abord il y a, au nom de l'assainissement des finances, l'orientation ambulatoire des services chirurgicaux. Ensuite, il y a le vieillissement des «baby boomers» dont les besoins chirurgicaux relèveront de plus en plus de services ambulatoires. Les données de Toronto, présentées par Chung et coll. ${ }^{1}$ donnent un intéressant aperçu de la perspective du vieillissement.

Les patients âgés ne représentent pas de risque supplémentaire dans l'environnement ambulatoire si l'on en juge par les mesures standard de qualité (mortalité et absence de complications graves) des soins anesthésiques monitorés (SAM). Ces patients éprouvent des dérangements physiologiques (cardiovasculaires surtout), liés au traumatisme chirurgical et à leur état de santé, mais cela ne semble pas les mettre en danger. Leur prise en charge fait partie du champ d'activités de tout anesthésiologiste. Certaines fonctions physiologiques sont fondamentalement instables sous anesthésie parce que beaucoup de réflexes nerveux qui modulent ces fonctions sont altérés par les anesthésiques. L'évaluation de la qualité d'un anesthésique basée sur la présence et l'importance de changements de TA, de FC et d'autres variables physiologiques est intéressante, mais ne repose sur aucune base scientifique. ${ }^{2}$ On doit démontrer clairement le lien entre la morbidité et ces changements physiologiques, et même alors, la fin (faible mortalité et morbidité) ne justifie pas les moyens (le coût prohibitif d'essais à grande échelle). En fait, le lien entre l'état de l'affection et les complications périopératoires est plus important. ${ }^{3}$ L'expérience pratique de l'anesthésiologiste est aussi à considérer. ${ }^{3}$ 
TABLE Taux de NVPO chez les patients ambulatoires de 1923 à 1998 (de l'éther au propofol). ${ }^{1-10}$

\begin{tabular}{lllll}
\hline Auteur (année) & $n$ & Taux de NVPO(\%) & $\begin{array}{l}\text { Technique } \\
\text { anesthésique }\end{array}$ \\
\hline Lundy (1923) & 500 & 25 & Ether, $\mathrm{N}_{2} \mathrm{O}, \mathrm{O}_{2}$ \\
Brindle (1975) & 500 & 16 & Halothane, $\mathrm{N}_{2} \mathrm{O}, \mathrm{O}_{2}$ \\
Duncan (1993) & 6914 & 7 & Isoflurane, $\mathrm{N}_{2} \mathrm{O}, \mathrm{O}_{2}$ \\
Chung (1996) & 1017 & 7 & Propofol, \\
& & & Isoflurane, $\mathrm{N}_{2} \mathrm{O}, \mathrm{O}_{2}$ \\
Chung (1998) & 17638 & 2 & Propofol, Isoflurane, \\
& & $\mathrm{N}_{2} \mathrm{O}, \mathrm{O}_{2}$ \\
\hline
\end{tabular}

Les résultats de l'enquête menée à Toronto permettent encore d'examiner d'autres sujets remis en question depuis longtemps. On s'attend souvent à ce que les anesthésiologistes admettent que la douleur et les vomissements sont des séquelles inévitables de leur pratique. Cependant, si l'anesthésique doit rétablir promptement les fonctions et les capacités en suspens, on ne devrait pas s'étonner que la présence de douleur et de vomissement soumette cet anesthésique au jugement de tous les patients qui continuent de souhaiter ardemment que leur soit évité ce genre de complications cliniques. ${ }^{4}$ Dans la série de Toronto, l'incidence des vomissements était de $2,7 \%$ avec l'anesthésie générale (AG) et de $0,6 \%$ chez les patients âgés qui ont reçu des SAM pour la plupart. Est-ce acceptable ? Des comparaisons historiques (Table) contribuent à démontrer que ces résultats sont excellents et que d'autres centres devraient viser une performance semblable. Peut-on y parvenir dans d'autres centres? Probablement. Edsall ${ }^{5}$ a montré qu'un programme trimestriel d'amélioration continue de la qualité (ACQ) qui s'est attaqué au problème des NVPO a été couronné de succès, réduisant le taux de $14 \%$ à $6 \%$ en huit mois. Les mesures comprennent l'usage réduit de narcotiques et de prétraitement chez les cas à risque.

Le traitement de la douleur et le rôle des opiacés en chirurgie ambulatoire sont d'autres aspects importants. C'est cruel de laisser un patient souffrir dans la salle de réveil, par manque de savoir-faire, dépendant de l'infirmière qui a comme premier recours une seringue d'opiacés. Avant la découverte de l'anesthésie, la prescription courante de laudanum visait à soulager la douleur de l'incision chirurgicale, mesure inutile puisque les patients le vomissaient. Déjà les médecins commençaient à se douter de cette action des opiacés sur le cerveau. Pourtant, aujourd'hui, nous utilisons le même remède analgésique. La série de Toronto démontre l'interaction entre douleur et opiacés. Après une AG chez de jeunes patients, le taux de NVPO à la salle de réveil est de $2,7 \%$, mais double presque à l'UCA (unité de chirurgie ambulatoire), résultat de l'administration d'opiacés à la salle de réveil. Rien d'étonnant à ce que même l'anesthésie au propofol soit d'une efficacité clinique douteuse, ce que montre l'analyse des NVPO de 84 études portant sur 6069 patients. ${ }^{6}$ Le meilleur anesthésique au propofol sera rendu inefficace par l'administration d'opiacés au patient à la salle de réveil. L'enquête de Toronto montre la possibilité d'un contrôle efficace de la douleur et une diminution de l'incidence de douleur nécessitant un traitement à la salle de réveil, la remenant à de très bas niveaux (6\%). C'est prometteur, car le traitement incomplet de la douleur chez les patients ambulatoires est un sujet permanent d'inquiétude. Dans les unités de soins ambulatoires qui n'utilisent que des opiacés, $40 \%$ des patients ont des douleurs modérées à sévères pendant l'hospitalisation. ${ }^{7}$ C'est inacceptable. L'enquête montre aussi que les SAM sont associés à de très faibles taux de douleur $(0,6 \%)$ et de vomissements $(0,6 \%)$ et, donc, à un séjour hospitalier écourté et à un risque réduit de réadmission. Pour l'anesthésie ambulatoire, il est fortement suggéré de remplacer les opiacés, entre autres par l'anesthésie multimodale avec l'emploi d'AINS et d'anesthésiques locaux.

En conclusion, c'est paradoxal de voir que les patients évaluent l'habileté d'un chirurgien d'après l'apparence de l'incision et que, de même ils évaluent l'anesthésique d'après l'absence de vomissements et de douleurs, et non par les obstacles invisibles qui ont été franchis pendant leur insensibilité. Il est nettement recommandé que l'organisation des services ambulatoires exige la mise en pratique de stratégies qui récoltent l'adhésion volontaire à un programme d'ACQ et vise à atteindre les données proposées comme références par la série de Toronto.

\section{«Étant jeune, répondit William à son fils, Je craignais qu cela ne nuisit au pensoir; Mais, désormais, convaincu de n'en point avoir, Je peux sans nul souci faire un tel exercice." \\ (Alice au Pays des Merveilles) \\ (Traduction de Henri Parisot)}

\section{References}

I Chung F, Mezei $G$, Tong D. Adverse events in ambulatory surgery: a closer look at the elderly. Can J Anesth 1999: 46: 309-21.

2 Coben MM, Duncan PG, Pope WDB, et al. The Canadian four-centre study of anaesthetic outcomes: II. Can outcomes be used to assess the quality of anesthetic care? Can J Anaesth 1992; 39: 430-9. 
3 Duncan PG, Cohen MM. Postoperative complications: factors of significance to anaesthetic practice. Can J Anaesth 1987; 34: 2-8.

4 Macario $A$, Weinger $M$, Carney $S$, et al. Which clinical anesthesia outcomes do patients find most undesirable? Anesthesiology 1998; 89: Al330.

5 Edsall DW. Utilizing information systems to facilitate performance based improvements in the management of postoperative nausea and vomiting in the ambulatory setting. American Society of Anesthesiologists, Annual Refresher Course Lectures 1998; 113: 1-7.

6 Tramer $M$, Moore $A, M c Q u a y$ H. Propofol anaesthesia and postoperative nausea and vomiting: quantitative systematic review of randomized controlled studies. $\mathrm{Br}$ J Anaesth 1997; 78: 247-55.

7 Beauregard L, Pomp A, Choinière $M$. Severity and impact of pain after day-surgery. Can J Anaesth 1998; 45: 304-11.

8 Marak B, Vasdev GM, Scott KP, et al. John Silas Lundy: the first auditor of anesthetic records. Anesthesiology 1998; 89: A94.

9 Brindle GF, Soliman $M G$. Anesthetic complicarions in surgical out-patients. Can J Anaesth $1975 ; 22$ : 613-9.

10 Chung $F$, Un V, Su J. Postoperative symptoms 24 hours after ambulatory anesthesia. Can J Anaesth 1996; 43: 1121-7. 